\title{
Role of the plain radiograph and urinalysis in acute ureteric colic
}

\author{
R Boyd, A J Gray
}

\begin{abstract}
Objective-(1) To determine the accuracy of accident and emergency (A\&E) doctors' diagnosis of radio-opaque ureteric calculi on plain abdominal radiographs; (2) to study the predictive value of haematuria with a history suggestive of ureteric colic. Design-A prospective study of all patients seen in a three month period with a provisional diagnosis of ureteric colic. Intravenous urography (IVU) was used as the gold standard for diagnosis of ureteric calculi.
\end{abstract}

Setting-The accident and emergency department and medical unit of a large teaching hospital.

Subjects -60 patients who were admitted with an initial diagnosis of ureteric colic, 51 subsequently undergoing intravenous urography.

Results-A\&E doctors achieved a calculated sensitivity of $29 \%$ ( $95 \%$ confidence intervals $13 \%$ to $49 \%$ ) and a specificity of $73 \%(52 \%$ to $90 \%)$ for identification of renal calculi on plain abdominal radiograph, compared with figures of $68 \%(48 \%$ to $84 \%)$ and $96 \%(78 \%$ to $100 \%)$ respectively for consultant radiologists. The difference between these results was highly significant $(P=0.0011)$. No patient with a definitive diagnosis of ureteric colic had a negative result for haematuria on urinary dipstick analysis.

Conclusions-A\&E doctors are poor at identifying radio-opaque ureteric calculi on plain abdominal radiographs. If haematuria is absent on urinalysis then ureteric colic is an unlikely diagnosis. ( $₹$ Accid Emerg Med 1996;13:390-391)

Key terms: ureteric colic; diagnosis; plain $x$ ray; urinalysis

\section{Department of}

Accident and

Emergency, Royal

Infirmary of

Edinburgh, Lauriston

Place, Edinburgh, EH3

9YW, United Kingdom

R Boyd

A J Gray

Correspondence to: Dr A J Gray, Research Registrar, Accident and Emergency Department, St James's University Hospital, Beckett Street, Leeds LS9 7TF.

Accepted for publication 17 July 1996

Renal colic caused by calculi is a common urological condition, occurring in approximately $2 \%$ of the western population, with $50 \%$ of these patients having recurrent episodes. These patients often present directly to accident and emergency (A\&E) departments and are seen initially by relatively inexperienced medical staff. There is frequently a classical combination of symptoms, often associated with microscopic or frank haematuria. The diagnosis is usually straightforward and is substantiated by a variety of radiological tests including plain abdominal films, ultrasonography, and intravenous urography. Intravenous urography is presently accepted as the gold standard investigation to confirm the clinical diagnosis during an acute episode. ${ }^{1-3}$ It is accepted practice to include the first of these tests-the plain abdominal film-as a useful and necessary adjunct to aid the early diagnosis of renal colic. ${ }^{3-5}$ Previous studies have suggested that up to $90 \%$ of all calculi are visualised on plain radiographs ${ }^{6}$ but other studies have given conflicting results. ${ }^{78}$

Only two studies have attempted to compare the presence of haematuria on urinary dipstick analysis against the findings of calculi on intravenous urography (IVU) in patients with acute ureteric colic. ${ }^{9} 10$ To date, the negative predictive value of dipstick urinalysis in the investigation of urinary calculi has not been estimated.

\section{Methods}

We studied 60 consecutive patients with a provisional diagnosis of acute ureteric colic admitted from the A\&E department of the Royal Infirmary of Edinburgh in the three months from August to October 1994.

Each patient was assessed initially by an A\&E doctor (SHO, registrar, or senior registrar with no formal radiological training). Plain abdominal radiographs and urinalysis with BoeringerMannheim dipsticks were performed before admission on all patients with an initial diagnosis of ureteric colic.

Every plain abdominal radiograph was reported as either positive or negative for radio-opaque calculi independently by both the $A \& E$ doctor and a consultant radiologist. Urinalysis was reported as positive or negative for blood. Lastly the intravenous urograms were reported as positive or negative for calculi by a consultant radiologist. The radiologists reported IVU and the plain abdominal films together. The abilities of the two groups of doctors in identifying calculi on plain films were compared using $\chi^{2}$ and McNemar's tests.

\section{Results}

Fifty one of the 60 patients subsequently underwent intravenous urography within 24 hours of admission. One patient was lost to follow up. The remaining eight patients were excluded from further analysis, as an alternative diagnosis was made soon after admission..

Table 1 compares A\&E doctors' plain abdominal film interpretation against the intravenous urograph (IVU) result.

This gives a calculated sensitivity of $29 \%$ (95\% confidence interval $13 \%$ to $49 \%$ ) with a specificity of $73 \%(52 \%$ to $90 \%)$ for the A\&E doctors' interpretation of the plain abdominal 
Table 1 AEE doctors' interpretation of the plain abdominal films

\begin{tabular}{lcc}
\hline & Positive IVU & Negative IVU \\
\hline Plain film positive & 8 & 6 \\
Plain film negative & 20 & 17 \\
\hline
\end{tabular}

Table 2 Consultant radiologists' interpretation of the plain abdominal films

\begin{tabular}{lcc}
\hline & Positive IVU & Negative IVU \\
\hline Plain film positive & 19 & 1 \\
Plain film negative & 9 & 22 \\
\hline
\end{tabular}

films when compared with IVU. The resultant positive predictive value of the $A \& E$ interpreted plain abdominal films for radio-opaque calculi was $57 \%$ ( $29 \%$ to $82 \%$ ).

The radiologists' plain abdominal film reports are compared with the IVU results in table 2. After comparison of the data a sensitivity for the radiologists' interpretation of $68 \%$ (48\% to $84 \%)$ and a specificity of $96 \%$ (78\% to $100 \%)$ were calculated. The positive predictive value for a radiologist reported plain abdominal film was therefore $95 \%$ ( $75 \%$ to $100 \%)$, with a negative predictive value of $71 \%(52 \%$ to $86 \%$ ).

$A \& E$ and radiology medical staffs' interpretational skills at identifying radio-opaque calculi were compared. A significant difference in skills was found $(P=0.0011)$ using $\mathrm{McNe}$ mar's and $\chi^{2}$ analysis.

The final comparison was made between urinalysis positive for haematuria and intravenous urography in table 3. This produced a sensitivity for a positive urinalysis result of $100 \%$ (88\% to $100 \%)$ and a specificity of $32 \%(21 \%$ to $73 \%)$. The positive predictive value of haematuria on dipstick was therefore $64 \%(50 \%$ to $80 \%)$ and a negative predictive value was $100 \%$ (59\% to $100 \%$ ).

\section{Discussion}

It is standard teaching and practice to include a plain abdominal radiograph in the initial investigation of acute ureteric colic This is based on the assumption that the majority of ureteric calculi are visualised. ${ }^{4}$ However, there is little objective evidence to support these conclusions. Two recent studies ${ }^{78}$ have suggested that plain radiographs are no better than clinical acumen at identifying patients with acute ureteric colic. Unfortunately all previous studies have involved interpretation of the plain abdominal radiographs by radiologists or urologists rather than by the doctor making the initial assessment in A\&E.

Our results for $A \& E$ doctors' interpretation of plain films are possibly poorer for a number of reasons. The training of $A \& E$ doctors is different from the doctors participating in previ-

Table 3 Haematuria compared with intravenous urography

\begin{tabular}{lll}
\hline & IVU positive & IVU negative \\
\hline Urinalysis positive for haematuria & 29 & 16 \\
Urinalysis negative for haematuria & 0 & 7 \\
\hline
\end{tabular}

ous studies. The plain radiographs may be of lower quality due to lack of bowel preparation and the patient's pain, resulting in movement artefact. Calculi may be too small to be identified on plain radiographs $(<2 \mathrm{~mm})$. Our patient population is likely to be different from those examined in other studies, which were retrospective and involved patients with a discharge diagnosis of ureteric colic rather than a presumptive admission diagnosis. However, despite this the need for improved teaching in the interpretation of plain radiographs among $\mathrm{A} \& \mathrm{E}$ staff is clear. Interestingly, the radiologists' reports from our study are better than from other recent studies. ${ }^{7} 8$ This may be because both investigations were reported simultaneously.

All 60 patients were admitted and underwent IVU independently of the plain radiograph result. This suggests that the plain film as a screening investigation should be stopped, since it entails significant costs and substantial radiation exposure with no obvious benefit. Most patients also had a second scout plain film at the time of the IVU to locate the approximate site of obstruction.

The use of the IVU as a gold standard is a potential limitation to the interpretation of our results. We did not allow for false negative IVU results, due to misinterpretation or to the passage of the calculus per urethra. Interobserver variabilities were also not taken into account.

Urinalysis proved to be sensitive but not particularly specific for the identification of calculi. However, it is certainly a far better screening investigation than plain radiographs, being safe, quick, and cheap.

\section{CONCLUSION}

The plain radiograph should not be used routinely in the A\&E department as a screening investigation for acute ureteric colic. The initial diagnosis should be made by eliciting an accurate history and by the results of urinalysis for haematuria. Subsequent confirmation of the diagnosis should be made by intravenous urography. We would like to thank Mr Bill Adams for advice on the interpretation of the statistics. We are grateful to
for her constant advice and for the use of SPSS.

1 RCR Working Party. Making the best use of a department of clinical radiology: guidelines for doctors. London: Royal College of Radiologists, 1995.

2 Webb JAW. Ultrasonography in the diagnosis of renal Webb JAW. Ultrasonography in the

3 Spencer J, Lindsell D, Mastorakou I. Ultrasonography compared with intravenous urography in the investigation of adults with haematuria. $B M \mathscr{F} 1990 ; 301: 1074-6$.

4 Brenner BM, Milford EL, Seifter JL. Urinary tract obstruction. In: Wilson JD, et al, eds. Harrison's principles of internal medicine, 12th ed. New York: McGraw-Hill, 1991:1207.

5 Abber JC, McAninch JW. Renal colic: emergency evaluation and management. Am $\mathcal{F}$ Emerg Med 1985;3:56-63.

6 Thornbury JR, Parker TW. Ureteral calculi. Semin Roentgenol 1982;17:133-59.

7 Mutgi A, Williams JW, Nettleman M. Utility of the plain abdominal roentgenogram. Arch Intern Med 1991;151: 1589-92.

8 Roth CS, Bowyer BA, Bowyer TH. Utility of the plain abdominal radiograph for diagnosing ureteral calculi. $A n n$ Emerg Med 1985;14:311-5.

9 Bishop NL. The influence of emergency urography and haematuria on the diagnosis of ureteric colic. Clin Radiol haematuria on the

10 Freeland P. No haematuria-no IVU. Irish $f$ Med Sci 1987; 156:270-1. 